\section{Análisis volumétrico de la orofaringe según la morfología craneofacial mediante la tomografía cone beam}

\section{Volumetric analysis of oropharynx according to craniofacial morphology using cone-beam tomography}

\begin{abstract}
Resumen
Objetivo. Analizar las tomografías computarizadas de haz cónico (CBCT), de sujetos con diferente biotipo facial y diferente patrón esquelético para comparar el volumen de la vía aérea orofaríngea. Métodos. El estudio fue de carácter descriptivo y transversal. La muestra estuvo formada por 55 tomografías cone beam pertenecientes a sujetos de 15 a 43 ańos. Las tomografías se agruparon según el biotipo facial, el patrón esquelético y por grupos etarios (15 - 20, 21 - 29, y 30 - 43 años). Se crearon cefalogramas virtuales para hallar el índice de Vert y el ángulo ANB. Los datos fueron analizados mediante las pruebas estadísticas: $U$ de Mann-Whitney, ANOVA y Kruskal-Wallis. Resultados. No se encontró una diferencia estadísticamente significativa entre el volumen orofaríngeo y el biotipo facial, ni entre el volumen orofaríngeo y el patrón esquelético. Se observó que los volúmenes más bajos fueron registrados en sujetos con patrón esquelético clase II $\left(\bar{x}=8,70 \pm 3,60 \mathrm{~cm}^{3}\right)$ y en sujetos con biotipo braquifacial $\left(\overline{\mathrm{x}}=8,87 \pm 2,96 \mathrm{~cm}^{3}\right)$. No hubo diferencia significativa entre el volumen y el sexo de los sujetos $(p>0,05)$. El grupo de 15 a 20 años presentó el mayor volumen orofaríngeo $\left(\bar{x}=11,16 \pm 5,64 \mathrm{~cm}^{3}\right)$. Adicionalmente, se registró una correlación negativa entre el volumen orofaríngeo y el ángulo ANB $(\mathrm{r}=-0,31, p=0,02)$. Conclusiones. El volumen orofaríngeo según el biotipo facial y el patrón esquelético no presenta una variación significativa para la muestra analizada. Además, el volumen orofaríngeo no varía de acuerdo al sexo, y es mayor entre los 15 y 20 ańos.
\end{abstract}

Palabras clave: Orofaringe; Maloclusión; Tomografía (fuente: DeCS BIREME).

\section{Artículo Original}

Héctor Miguel Huamaní Guzman 1,a, Luciano Soldevilla Galarza $^{2, b}$, Aron Aliaga-Del Castillo ${ }^{3, c}$

${ }^{1}$ Universidad Nacional Mayor de San Marcos, Facultad de Odontología, Lima, Perú.

2 Universidad Nacional Mayor de San Marcos, Facultad de Odontología, Departamento Académico de Estomatología Pediátrica, Lima, Perú.

${ }^{3}$ Universidade de São Paulo, Faculdade de Odontologia de Bauru, São Paulo, Brasil.

a Cirujano Dentista.

${ }^{\mathrm{b}}$ Especialista en Ortodoncia

${ }^{c}$ Magíster en Ortodoncia.

\section{Correspondencia:}

Héctor Miguel Huamaní Guzman: hhuamaniguzman@ gmail.com

Av. José Carlos Mariátegui $\mathrm{N}^{\circ} 287$, Sector A, Villa María del Triunfo, Lima, Perú.

ORCID: 0000-0003-2954-029X

\section{Coautores:}

Luciano Soldevilla Galarza: Isoldevillag@unmsm.edu.pe ORCID: 0000-0002-5939-4644

Aron Aliaga-Del Castillo: a_aliaga@hotmail.com

ORCID: 0000-0003-3963-1742

\section{Editora:}

Antonieta Pérez-Flores

Universidad de Concepción, Chile.

Conflicto de intereses: los autores declaran no tener ningún conflicto de interés en la publicación del artículo.

Fuente de financiamiento: autofinanciado.

\section{Recibido: 23/05/20}

Aceptado: 04/08/20

Publicado: $16 / 11 / 20$

(C) Los autores. Este artículo es publicado por la revista Odontología Sanmarquina de la Facultad de Odontología, Universidad Nacional Mayor de San Marcos. Este es un artículo de acceso abierto, distribuido bajo los términos de la licencia Creative Commons Atribucion - No Comercia_Compartir Igual 4.0 Internacional. (http://creativecommons.org/licenses/by-nc-sa/4.0/) que permite el uso no comercial, distribución y reproducción en cualquier medio, siempre que la obra original sea debidamente citada. 
to find the Vert index and the ANB angle. Data were analyzed using statistical tests: $U$ of Mann-Whitney, ANOVA, and Kruskal-Wallis. Results. No statistically significant difference was found between oropharyngeal volume and facial biotype, nor between oropharyngeal volume and skeletal pattern. The lowest volumes were identified in subjects with class II skeletal pattern $\left(\bar{x}=8.70 \pm 3.60 \mathrm{~cm}^{3}\right)$, and in subjects with brachyfacial biotype $(\overline{\mathrm{x}}=$ $\left.8.87 \pm 2.96 \mathrm{~cm}^{3}\right)$. There was no significant difference between volume and sex $(p>0.05)$. The 15-20-year-old group presented the highest volume of oropharyngeal $(\bar{x}=11.16 \pm 5.64$ $\left.\mathrm{cm}^{3}\right)$. Also, a negative correlation was recorded between oropharyngeal volume and the ANB angle $(r=-0.31, p=0.02)$. Conclusions. Oropharyngeal volume according to facial biotype and the skeletal pattern does not present a significant variation for the analyzed sample. Also, the oropharyngeal volume does not vary according to sex, and the volume is greater between 15 and 20 years.

Keywords: Oropharynx; Malocclusion; Tomography (source: MeSH NLM).

\section{Introducción}

La relación entre la morfología craneofacial y la función respiratoria ha sido el centro de muchas investigaciones desde el siglo XIX. El estudio de las estructuras de la vía aérea respiratoria incluye los trabajos de Linder-Aronson ${ }^{1}$ sobre el crecimiento mandibular en pacientes sometidos a una adenoidectomía, los trabajos de Solow y su hipótesis del estiramiento de los tejidos blandos ${ }^{2}$, hasta los recientes estudios acerca de la morfología de la vía aérea asociada con el síndrome de apnea obstructiva del sueño (SAOS) ${ }^{3}$.

A lo largo de la historia, muchos estudios han empleado la cefalometría lateral como principal medio para evaluar la vía aérea superior, y determinar si existe una relación entre el tamaño de la nasofaringe y la orofaringe con el patrón esquelético ${ }^{4,5}$. Sin embargo, este instrumento presenta grandes limitaciones como: la representación bidimensional (2D) de una estructura que es tridimensional (3D), la magnificación y la superposición de estructuras bilaterales ${ }^{6}$.

Por otro lado, la tomografía computarizada cone beam (CBCT) fue desarrollada a finales de los años noventa ${ }^{7}$, y se a convertido en la mejor herramienta para el análisis de estructuras tridimensionales ${ }^{8}$ como la vía aérea superior. La CBCT permite obtener imágenes de alta definición con menor tiempo de adquisición y menor dosis de radiación que la tomografía computarizada convencional. Además, permite la manipulación multiplanar de imágenes, la medición longitudinal y volumétrica de estructuras y su reconstrucción 3D 9,10 . También, cuenta con la opción para generar cefalogramas virtuales y poder realizar mediciones cefalométricas en ellos ${ }^{11,12}$.

Precisamente es la CBCT la que hoy en día nos permite conocer que existen una serie de problemas que tienen lugar en la orofaringe; por ejemplo, la hipertrofia de las amígdalas, que puede inducir el posicionamiento anterior de la lengua, aumentar el espacio orofaríngeo y agravar algunas maloclusiones como la mordida abierta anterior ${ }^{13}$. Caso contrario sucede con la disminución del espacio orofaríngeo, que puede estar asociada con el SAOS y provocar una facies adenoidea, un plano mandibular más inclinado y una posición más inferior del hueso hioides ${ }^{14,15}$.
En este contexto, existen varios trabajos que han buscado relacionar el tamaño de la vía aérea respiratoria superior y su influencia en el crecimiento y desarrollo craneofacial. Recientemente, ha habido un aumento en el número de estudios que emplean el desarrollo de imágenes 3D para la evaluación de la orofaringe mediante la CBCT. Sin embargo, en el Perú, la evidencia de este tipo de trabajos es escasa. Por ello, el propósito de este estudio fue comparar el volumen de la vía aérea orofaríngea según el biotipo facial y el patrón esquelético utilizando la CBCT.

\section{Metodos}

Este estudio fue de carácter descriptivo y de corte transversal, y contó con la aprobación del comité de ética del Instituto de Diagnóstico Maxilofacial (IDM), Lima, Perú. Se recolectaron 115 CBCT de pacientes que acudieron al IDM desde setiembre del 2013 hasta enero del 2015. Las tomografías fueron adquiridas con una unidad Planmeca ProMax 3D Mid, con la siguiente configuración: $90 \mathrm{Kv}, 10 \mathrm{~mA}$, tiempo de exposición de 13.68 segundos, tamaño de voxel de $400 \mu \mathrm{m}$, campo de visión de $23 \times 17 \mathrm{~cm}$ y un formato de imagen DICOM (digital imaging and communications in medicine).

Muesta de estudio. La muestra inicial estuvo formada por tomografías de sujetos, de 15 a 43 años de edad, cuyo tamaño abarcara desde el punto Trichion hasta el borde inferior de la cuarta vértebra cervical (campo visual grande). Durante la evaluación de las tomografías, se excluyeron aquellas que presentaban evidencia de tratamiento ortodóntico (brackets), cirugía ortognática (miniplacas), y obstrucción de la vía aérea orofaríngea. La muestra final estuvo formada por 55 tomografías, de 22 hombres y 33 mujeres (edad media de 25,78 años), que se agruparon según el biotipo facial, patrón esquelético y sexo. Además, se formaron tres grupos etarios: de 15 a 20 años, de 21 a 29 años y de 30 a 43 años.

Todas las mediciones fueron realizadas por un mismo investigador, quien previamente fue sometido a una calibración para determinar la ubicación exacta de los puntos cefalométricos en el software Planmeca Romexis Viewer 3.5.1. La calibración estuvo a cargo de un docente de la especialidad de Ortodoncia de la 
Facultad de Odontología de la Universidad Nacional Mayor de San Marcos. Para ello, se analizaron seis tomografías (10\% de la muestra) elegidas aleatoriamente y se utilizó el índice Kappa de Cohen, el valor obtenido para la determinación del biotipo facial fue 0,75 (75\%) mientras que para el patrón esquelético fue 1,0 $(100 \%)$
Análisis cefalométrico. Se creó un cefalograma virtual a partir de cada CBCT. Se ajustó la calidad de imagen y el grado de nitidez para mejorar la visibilidad. Luego, se ubicaron los puntos cefalométricos para calcular el índice de Vert (IV) y medir el ángulo ANB (Figura 1). La Tabla 1 describe los planos y ángulos utilizados en el análisis cefalométrico.

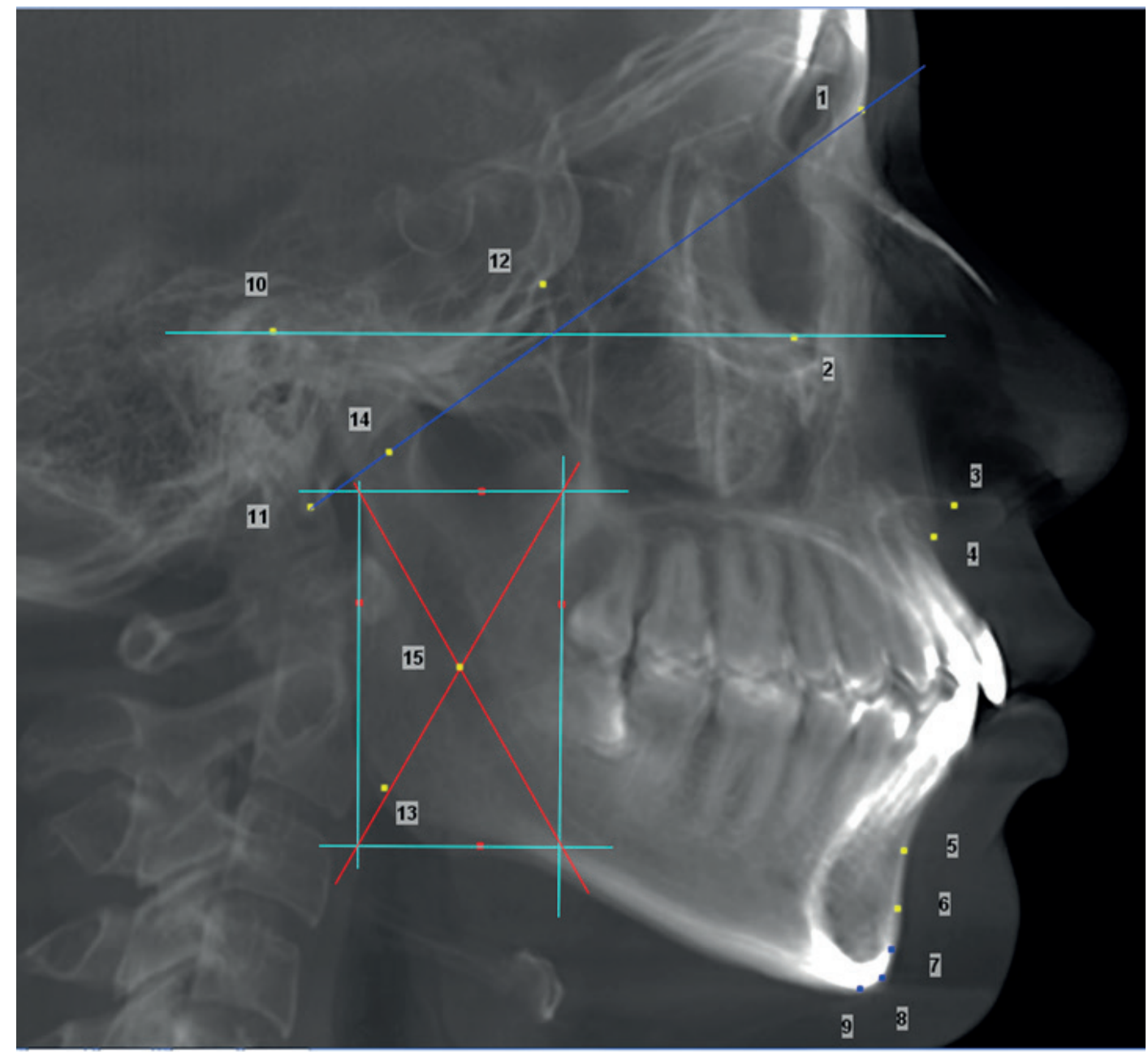

Figura 1. Ubicación de los puntos cefalométricos. 1- N (Nasion), 2- Or (Orbital), 3- ENA (Espina nasal anterior), 4-punto A, 5- punto B, 6- Pm (Protuberancia menti), 7- Pg (Pogonion), 8- Gn (Gnation), 9- Me (Mentoniano), 10- Po (Porion), 11- Ba (Basion), 12- Pt (Pterigoideo), 13- Go (Gonion), 14- Dc (Cuello condilar), 15- Xi (Centro de rama mandibular)

Tabla 1. Definición de los planos y ángulos cefalométricos utilizados en el estudio

\begin{tabular}{ll}
\hline \multicolumn{1}{c}{ Ángulo } & \multicolumn{1}{c}{ Definición } \\
\hline Eje facial & Formado por los planos Ba-N y Pt-Gn \\
Profundidad facial & Formado por los planos N-Gn y Po-Or (Plano de Frankfort) \\
Ángulo del plano mandibular & Formado por los planos Po-Or y Go-Me \\
Altura facial inferior & Formado por la línea Xi-ENA con el eje del cuerpo mandibular (Xi-Pm) \\
Arco mandibular & Formado por el eje condilar (Dc-Xi) con la prolongación distal de Xi-Pm \\
ANB & Formado por los planos N-A y N-B \\
\hline
\end{tabular}

Tomado de: Gregoret ${ }^{16}$ y Zamora-Montes de Oca ${ }^{17}$ 
Los rangos para clasificar el biotipo facial fueron: $I V \leq-$ 0,5 , biotipo dolicofacial, $-0,5<\mathrm{IV}<0,5$, biotipo mesofacial y IV $\geq 0,5$, biotipo braquifacial ${ }^{16}$. Para determinar el patrón esquelético se empleó el ángulo ANB. Si $\mathrm{ANB}<0^{\circ}$, el patrón era Clase III, si $0^{\circ} \leq \mathrm{ANB} \leq 4^{\circ}$, el patrón era Clase I y si $\mathrm{ANB}>4^{\circ}$, el patrón era Clase II ${ }^{17}$.

Volumen orofaríngeo. Se reorientó la posición de la cabeza para estandarizar el procedimiento y minimizar los errores. Las vistas sagital y coronal se rotaron hasta que los puntos Orbital de ambos lados y el Porion derecho formen un único plano horizontal, llamado Frankfort
Horizontal $(\mathrm{FH})^{18}$. Además, se rotó la vista axial para alinear los puntos ENA y ENP (Figura 2).

Para calcular la vía orofaríngea se adoptaron los limites propuestos por El y Palomo ${ }^{19}$ : el límite superior fue el plano palatino (PP) y su extensión hasta la pared posterior faríngea; el límite inferior fue el plano paralelo a PP que pasa por el punto más anterior e inferior de la segunda vértebra cervical (2vc); el límite anterior fue el plano frontal que pasa por el punto ENP; el límite posterior fue la pared posterior faríngea; y los límites laterales fueron las paredes laterales de la faringe (Figura 3).

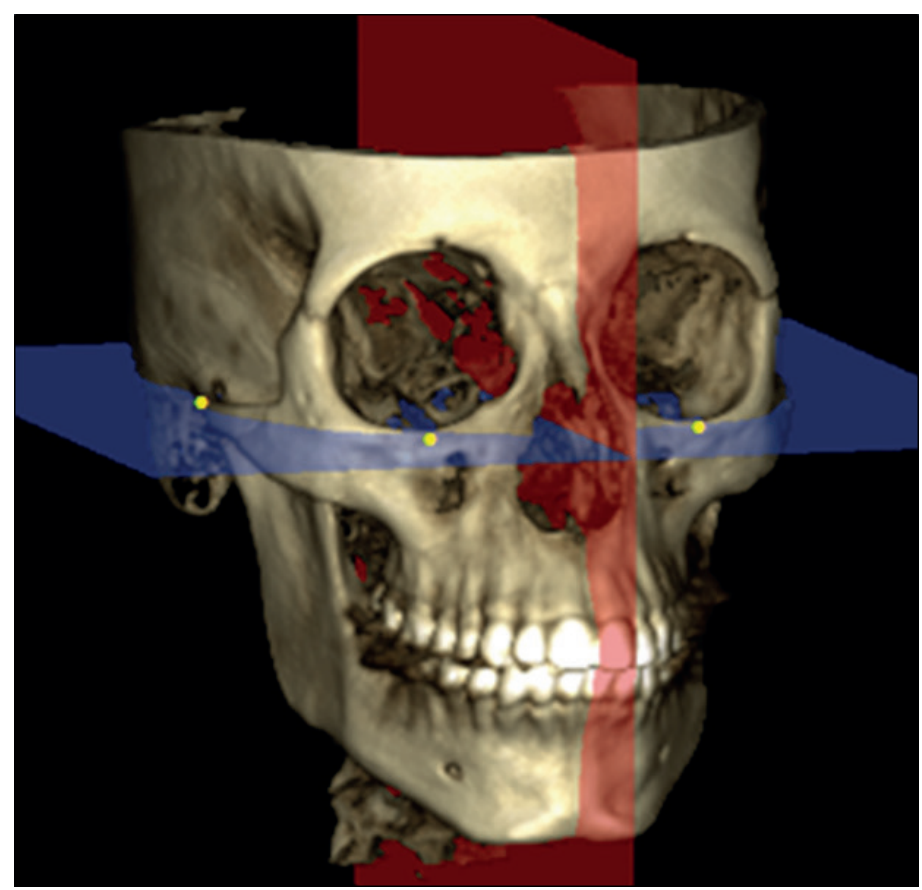

Figura 2. Orientación final de la cabeza. Alineación de los puntos Or de ambos lados y Po derecho, FH (plano azul). Alineación de ENA y ENP perpendicular a FH (plano rojo)

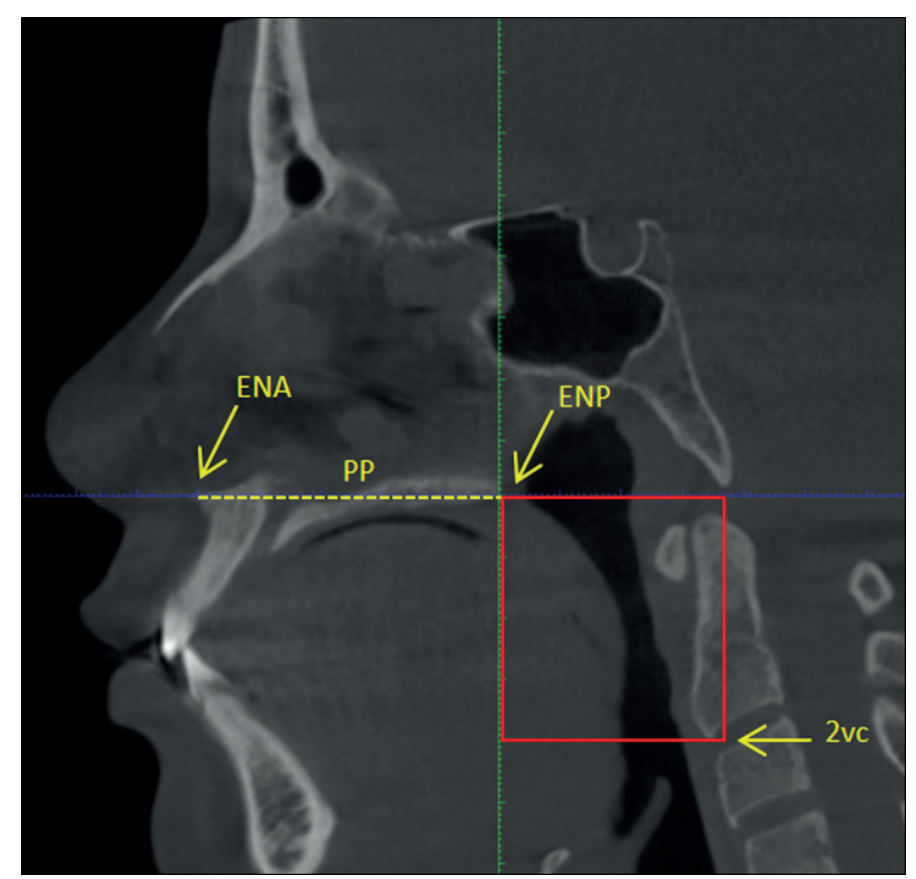

Figura 3. Delimitación de la vía orofaríngea, vista sagital 
Una vez delimitada la vía orofaríngea, se midió la región de crecimiento 3D de la cavidad aérea seleccionada para obtener el volumen en $\mathrm{cm}^{3}$ (Figuras 4 y 5 ).

El análisis estadístico se realizó en el programa SPSS 21 para Windows (IBM SPSS, Chicago, Illinois, USA). Se empleó la prueba estadística ANOVA para evaluar las variables biotipo facial y grupo etario. El patrón esquelético se analizó con la prueba Kruskal-Wallis, y el sexo, mediante la prueba t. Además, se utilizó la correlación de Spearman para determinar la relación entre el volumen orofaríngeo (VO) y los ángulos: eje facial, profundidad facial, plano mandibular y ANB. El nivel de significancia establecido fue del $5 \%$ para todas las pruebas.

\section{Resultados}

La distribución de la CBCT según el sexo, grupo etario, biotipo facial y patrón esquelético se puede observar en la Tabla 2.

El mayor VO se obtuvo en sujetos con biotipo mesofacial, mientras que el menor valor en sujetos con biotipo braquifacial (Tabla 3). Las tomografías de sujetos

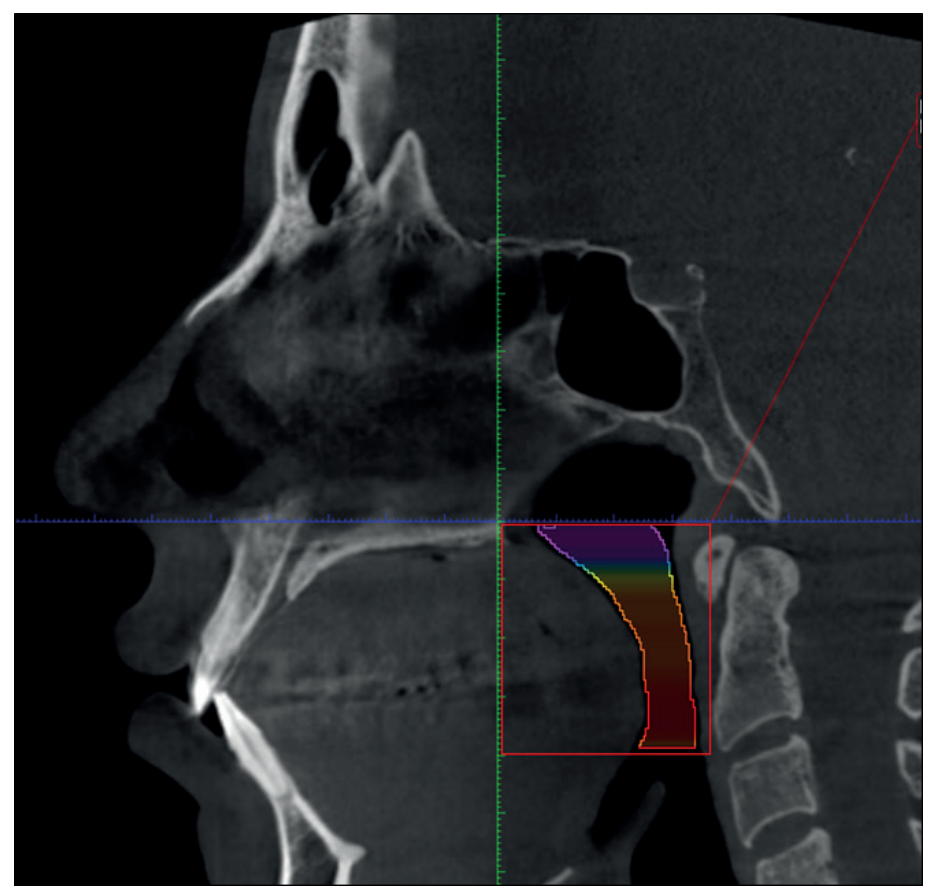

Figura 4. Región de crecimiento 3D de la vía orofaríngea, vista sagital

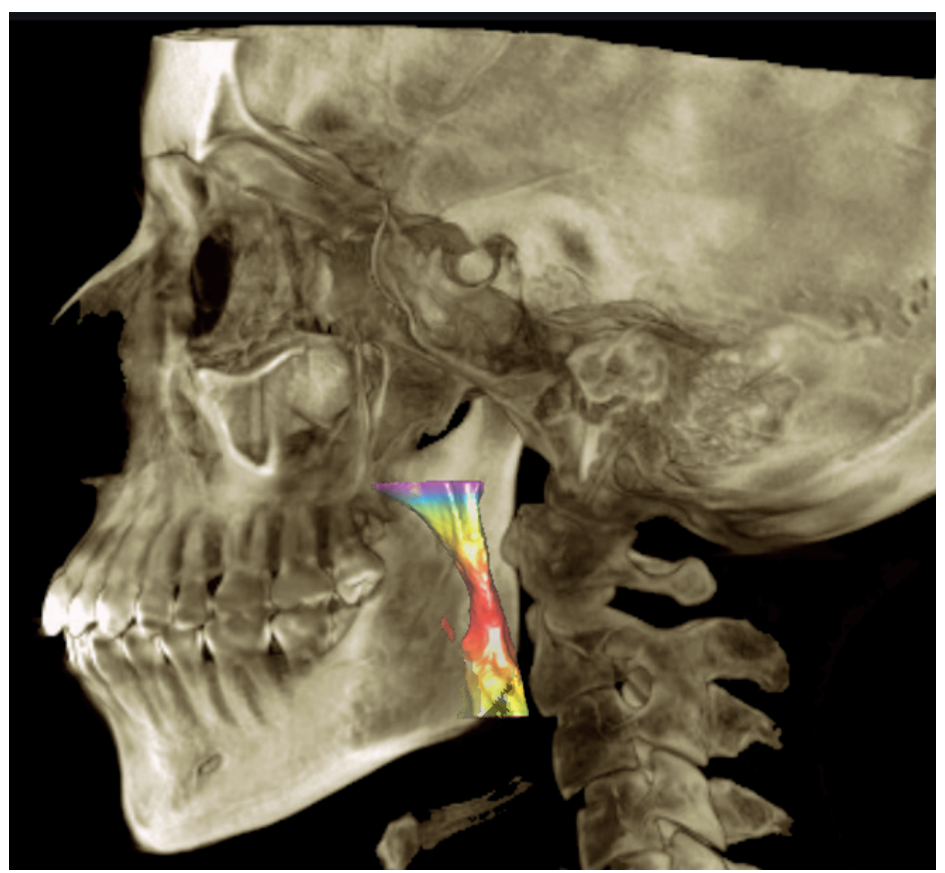

Figura 5. Región de crecimiento 3D de la vía orofaríngea en una reconstrucción tomográfica, vista sagital 
Tabla 2. Distribución de tomografías cone beam según el género, grupo etario, biotipo facial y patrón esquelético.

\begin{tabular}{|c|c|c|c|}
\hline \multicolumn{2}{|c|}{ Variables } & \multirow{2}{*}{$\begin{array}{c}\mathbf{n} \\
22\end{array}$} & \multirow{2}{*}{$\begin{array}{l}\mathbf{( \% )} \\
40,0\end{array}$} \\
\hline Sexo & Masculino & & \\
\hline & Femenino & 33 & 60,0 \\
\hline Grupo & $15-20$ & 13 & 23,6 \\
\hline \multirow[t]{2}{*}{ etario } & $21-29$ & 29 & 52,7 \\
\hline & $30-43$ & 13 & 23,6 \\
\hline Biotipo & Dolicofacial & 20 & 36,4 \\
\hline \multirow[t]{2}{*}{ Facial } & Mesofacial & 12 & 21,8 \\
\hline & Braquifacial & 23 & 41,8 \\
\hline Patrón & Clase I & 19 & 34,5 \\
\hline \multirow[t]{2}{*}{ Esquelético } & Clase II & 32 & 58,2 \\
\hline & Clase III & 4 & 7,3 \\
\hline
\end{tabular}

Tabla 3. Volumen orofaríngeo según el biotipo facial

\begin{tabular}{lccccccc}
\hline & $\mathbf{n}$ & $\begin{array}{c}\overline{\mathbf{x}} \\
\left(\mathrm{cm}^{3}\right)\end{array}$ & D. S. & Mín. & Máx. & \multicolumn{2}{c}{ IC (95\%) } \\
& 20 & 9,45 & 4,20 & 5,22 & 21,52 & 7,49 & 11,42 \\
Dolicofacial & 12 & 10,02 & 3,72 & 6,90 & 19,90 & 7,65 & 12,38 \\
Mesofacial & 23 & 8,87 & 2,96 & 3,09 & 13,98 & 7,59 & 10,15 \\
Braquifacial & &
\end{tabular}

$p=0,664$

con patrón esquelético clase I registraron el mayor $\mathrm{VO}$ y los sujetos clase II obtuvieron el menor valor (Tabla 4). Las pruebas estadísticas ANOVA y Kruskal-Wallis no indicaron diferencias significativas para el biotipo y el patrón esquelético respectivamente.

El VO promedio para los hombres fue de 9,30 $\pm 3,91$ $\mathrm{cm}^{3}$ y para las mujeres $9,36 \pm 3,39 \mathrm{~cm}^{3}$. La prueba $T$ indicó que no existe una diferencia significativa entre ambos sexos. Por otro lado, el mayor VO lo registró el grupo de 15 a 20 años $\left(11,16 \pm 5,64 \mathrm{~cm}^{3}\right)$, seguido por el grupo de 30 a 43 años $\left(8,98 \pm 2,49 \mathrm{~cm}^{3}\right)$ y el grupo de 21 a 29 años $\left(8,67 \pm 2,51 \mathrm{~cm}^{3}\right)$. Estos volúmenes no registraron una diferencia estadísticamente importante en la prueba ANOVA.

Al relacionar el VO con los ángulos eje facial, profundidad facial, plano mandibular y ANB, únicamente se obtuvo una correlación negativa con ángulo el ANB (Tabla 5).

\section{Discusión}

La teoría de la matriz funcional desarrollada por Moss establece que la mayor parte del crecimiento y desarrollo de la región craneofacial está bajo el control de la actividad funcional de los tejidos blandos circundantes. Por lo tanto, existe una interacción directa entre los espacios de las vías respiratorias faríngeas y la configuración craneofacial, de modo que cualquier anomalía en estos espacios puede afectar la posición del esqueleto circundante ${ }^{20}$.

La cuantificación de la vía aérea orofaríngea y la evaluación de la morfología 3D mediante exploraciones
CBCT proporciona un mejor sustituto de la evaluación radiográfica $2 \mathrm{D}$ convencional y ofrece un análisis preciso en los tres planos anatómicos ${ }^{21}$. El presente estudio realizó todas las mediciones angulares en cefalogramas virtuales generados a partir de CBCT. Este tipo de cefalogramas fue estudiado por Cattaneo et al. ${ }^{11} \mathrm{y}$ Kumar et al. ${ }^{12}$, quienes concluyeron que es posible utilizarlos para realizar los análisis cefalométricos con una precisión similar a la de los cefalogramas físicos.

Brasil et al. ${ }^{22}$ concluyeron que el tipo facial esquelético anteroposterior y el patrón esquelético no parecen afectar el volumen de la vía orofaríngea, la cual fue delimitada por un plano que iba desde la ENP hasta el punto más inferior de la vértebra Atlas (límite superior) y un plano tangente al punto más anteroinferior de la tercera vértebra cervical (límite inferior). En el presente trabajo tampoco se encontró una diferencia significativa al comparar el VO según el biotipo facial y el patrón esquelético. No obstante, para este trabajo, se consideraron otros límites para la vía orofaríngea y una muestra más pequeña.

Grauer et al. ${ }^{23}$ evaluaron la forma y el volumen de la vía aérea en sujetos con diferentes patrones faciales, y concluyeron que el volumen y la forma varían en pacientes con relaciones anteroposteriores diferentes; sin embargo, cuando se trata de relaciones verticales, solo varía la forma. En este estudio, el patrón clase II obtuvo el menor VO, mientras que la clase I y la clase III registraron valores similares. Estos resultados concuerdan con los estudios realizados por El y Palomo ${ }^{19}$ y con el reporte de Alves ${ }^{24}$. 
Tabla 4. Volumen orofaríngeo según el patrón esquelético

\begin{tabular}{lccccccc}
\hline & $\mathbf{n}$ & $\begin{array}{c}\overline{\mathbf{x}} \\
\left(\mathrm{cm}^{3}\right)\end{array}$ & D. S. & Mín. & Máx. & \multicolumn{2}{c}{ IC 95\% } \\
\hline Clase I & 19 & 10,24 & 3,72 & 5,62 & 21,52 & 8,45 & 12,04 \\
Clase II & 32 & 8,70 & 3,60 & 3,09 & 19,90 & 7,40 & 9,99 \\
Clase III & 4 & 10,11 & 1,59 & 8,53 & 11,93 & 7,57 & 12,65 \\
\hline
\end{tabular}

$p=0,147$

Tabla 5. Correlación de Spearman entre el volumen orofaríngeo y medidas angulares*

\begin{tabular}{lcccc}
\hline & EF & PF & PM & ANB \\
\hline Volumen orofaríngeo & 0,148 & 0,027 & $-0,066$ & $-0,313^{* *}$ \\
\hline
\end{tabular}

*EF indica eje facial; PF, profundidad facial; PM, plano mandibular.

${ }^{* *} p<0,05$

Daniel et al. ${ }^{25}$ analizaron el espacio de las vías respiratorias orofaríngeas de 10 hombres y 10 mujeres, entre las edades de 7 y 11 años, revelando que las dimensiones de las vías respiratorias son mayores en los hombres en comparación con las mujeres. Otros autores como Grauer et al. ${ }^{23}$, Claudino et al. ${ }^{26}$ y Shigetta et al. ${ }^{27}$, también hallaron que el volumen de la vía aérea fue significativamente mayor en los hombres. Sin embargo, El y Palomo ${ }^{28}$ no informaron tal distinción de sexo en términos de volumen de las vías respiratorias. Del mismo modo, el estudio actual no encontró una diferencia significativa entre el sexo y el VO, lo cual puede deberse al tamaño y distribución de la muestra empleada.

La literatura afirma que las estructuras de la vía faríngea continúan creciendo rápidamente hasta los 13 años de edad, y que luego ocurre un periodo de latencia hasta los 18 años ${ }^{29}$. En estudios longitudinales, se ha establecido que entre los 20 y 50 ańos de edad el paladar blando se extiende y aumenta su espesor, mientras que la región faríngea sufre un estrechamiento y un aumento de su longitud ${ }^{30}$. Ésta podría ser la razón por la que el mayor VO que se registró en el grupo etario de 15 a 20 años.

Ceylan y Oktay ${ }^{4}$ informaron que los cambios en el ángulo ANB afectaron no solo al tamaño de la vía aérea nasofaríngea, sino también al área orofaríngea, que se redujo en sujetos con un ángulo ANB aumentado. Claudino et al. ${ }^{26}$ encontraron una tendencia a la disminución del volumen de la porción inferior de la vía faríngea conforme aumentaba el ángulo ANB. Esto concuerda con la correlación negativa hallada en este trabajo entre el ángulo ANB y el VO.

Este estudio buscó comparar el VO entre diferentes biotipos faciales y patrones esqueléticos mediante la CBCT, debido a que son pocos los estudios realizados en el Perú que abordan este tipo análisis, por la importancia que tiene el uso de la CBCT como una evaluación alternativa para el diagnóstico del SAOS relacionado con la morfología de la clase II, y por ser fundamental en la planificación de un tratamiento ortodóntico o una cirugía ortognática.
Sin embargo, tuvo como limitación principal no disponer de una mayor cantidad de CBCT para una mejor distribución de la muestra; por ello, la diferencia entre los volúmenes no resultó estadísticamente significativa.

Este estudio no halló una variación relevante del VO de acuerdo al biotipo facial, ni al patrón esquelético de la muestra evaluada. Además, el VO no registró una diferencia importante según el sexo, y tuvo un mayor valor en sujetos entre los 15 y 20 años de edad. Se sugiere realizar nuevos estudios con una mayor muestra para llegar a una conclusión más sólida y representativa.

\section{Referencias bibliográficas}

1. Linder-Aronson S. Adenoids. Their effect on mode of breathing and nasal airflow and their relationship to characteristics of the facial skeleton and the dentition. A biometric rhino-manometric and cephalometro-radiographic study on children with and without adenoids. Acta Otolaryngol Suppl. 1970;265:1-132.

2. Di Carlo G, Polimeni A, Melsen B, Cattaneo PM. The relationship between upper airways and craniofacial morphology studied in 3D. A CBCT study. Orthod Craniofac Res. 2015;18:1-11. DOI:10.1111/ocr.12053

3. Chaudhry U, Cohen JR, Al-Samawi Y. Use of cone beam computed tomography imaging for airway measurement to predict obstructive sleep apnea. CRANIO ${ }^{\oplus}$. 2020:1-7. DOI:10.1080/08869634.2020.1765602

4. Ceylan I, Oktay H. A study on the pharyngeal size in different skeletal patterns. Am J Orthod Dentofacial Orthop. 1995;108(1):69-75. DOI:10.1016/S08895406(95)70068-4

5. De Freitas MR, Alcazar NM, Janson G, De Freitas KM, Henriques JF. Upper and lower pharyngeal airways in subjects with Class I and Class II malocclusions and different growth patterns. Am J Orthod Dentofacial Orthop. 2006;130(6):742-745. DOI:10.1016/j.ajodo.2005.01.033

6. Major MP, Flores-Mir C, Major PW. Assessment of lateral cephalometric diagnosis of adenoid hypertrophy and posterior upper airway obstruction: a systematic review. 
Am J Orthod Dentofacial Orthop. 2006;130(6):700708. DOI:10.1016/j.ajodo.2005.05.050

7. Lenguas A, Ortega R, Samara G, López M. Tomografía computarizada de haz cónico. Aplicaciones clínicas en odontología; comparación con otras técnicas. Cient Dent. 2010;7(2):147-59.

8. Lagravère MO, Carey J, Toogood RW, Major PW. Three-dimensional accuracy of measurements made with software on cone-beam computed tomography images. Am J Orthod Dentofacial Orthop. 2008;134(1):112-16. DOI:10.1016/j.ajodo.2006.08.024

9. Guijarro-Martínez R, Swennen GRJ. Cone-beam computerized tomography imaging and analysis of the upper airway: a systematic review of the literature. Int J Oral Maxillofac Surg. 2011;40(11):1227-37. DOI: 10.1016/j.ijom.2011.06.017

10. Ghoneima A, Kula K. Accuracy and reliability of cone-beam computed tomography for airway volume analysis. Eur J Orthod. 2011;35(2):256-261. DOI:10.1093/ ejo/cjr099

11. Cattaneo PM, Bloch CB, Calmar D, Hjortshoj M, Melsen B. Comparison between conventional and cone-beam computed tomography-generated cephalograms. Am J Orthod Dentofacial Orthop. 2008;134(6):798-802. DOI:10.1016/j.ajodo.2008.07.008.

12. Kumar V, Ludlow J, Mol A, Cevidanes L. Comparison of conventional and cone beam CT synthesized cephalograms. Dentomaxillofacial Radiology. 2007;36(5):263269. DOI: $10.1259 / \mathrm{dmfr} / 98032356$

13. Alcazar NM, De Freitas MR, Janson G, Henriques JF, De Freitas KM. Estudo cefalométrico comparativo dos espaços naso e buco faríngeo na más oclusôes Classe I e Classe II, divisão 1, sem tratamento ortodôntico, com diferentes padrôes de crescimento. Rev Dent Press Ortodon Ortop Facial. 2004;9(4):68-76. DOI:10.1590/ S1415-54192004000400007.

14. Nuernberg CH, Vilella OD. Avaliação cefalométrica da orofaringe. Rev Odonto Ciência. 2006;21(54):370-75.

15. Baik UB, Suzuki M, Ikeda K, Sugawara J, Mitani H. Relationship between cephalometric characteristics and obstructive sites in obstructive sleep apnea syndrome. Angle Orthod. 2002;72:124-134. DOI:10.1043/00033219

16. Gregoret J, Tuber E, Escobar LH, Matos A. Ortodoncia y Cirugía Ortognática: Diagnóstico y Planificación. Barcelona: Espaxs Editores; 1997. p. 227-232.

17. Zamora C. Compendio de cefalometría, análisis clínico y práctico. México: Amolca Editores; 2004. p. 178.

18. Oh KM, Kim MA, Youn JK, Cho HJ, Park YH. Three-dimensional evaluation of the relationship between nasopharyngeal airway shape and adenoid size in children. Korean J Orthod. 2013;43(4):160-67. DOI:10.4041/ kjod.2013.43.4.160

19. El H, Palomo JM. Airway volume for different dentofacial skeletal patterns. Am J Orthod Dentofacial Orthop. 2011;139(6):511-21. DOI:10.1016/j.ajodo.2011.02.015
20. Alhammadi MS, Almashraqi AA, Helboub E, Almahdi S, Jali T, Atafi A, et al. Pharyngeal airway spaces in different skeletal malocclusions: a CBCT 3D assessment. Cranio. 2019:1-10. DOI:10.1080/08869634.2019.158 3301

21. Tikku T, Khanna R, Sachan K, Agarwal A, Srivastava K, Lal, A. Dimensional and volumetric analysis of the oropharyngeal region in obstructive sleep apnea patients: A cone beam computed tomography study. Dent Res J. 2016;13(5):396.

22. Brasil DM, Kurita LM, Groppo FC, Haiter-Neto F Relationship of craniofacial morphology in 3-dimensional analysis of the pharynx. Am J Orthod Dentofacial Orthop. 2016;149(5):683-91. DOI:10.1016/j.ajodo.2015.11.021

23. Grauer D, Cevidanes LS, Styner MA, Ackerman JL, Proffit WR. Pharyngeal airway volume and shape from cone-beam computed tomography: relationship to facial morphology. Am J Orthod Dentofacial Orthop. 2009;136(6):805-14. DOI:10.1016/j.ajodo.2008.01.020

24. Alves M, Franzotti E, Baratieri C, Nunes L, Nojima L, Ruellas A. Evaluation of pharyngeal airway space amongst different skeletal patterns. International Journal of Oral and Maxillofacial Surgery. 2012;41(7):814-9. DOI:10.1016/j.ijom.2012.01.015

25. Daniel MM, Lorenzi MC, Leite CD, et al. Pharyngeal dimensions in healthy men and women. Clinics. 2007;62(1):5-10. DOI:10.1590/S180759322007000100002

26. Claudino LV, Mattos CT, de Oliveira Ruellas AC, Sant'Anna EF. Pharyngeal airway characterization in adolescents related to facial skeletal pattern: a preliminary study. Am J Orthod Dentofacial Orthop. 2013;143(6):799-809. DOI:10.1016/j.ajodo.2013.01.015

27. Shigeta Y, Ogawa T, Venturin J, Nguyen M, Clark GT, Enciso R. Gender and age based differences in computerized tomographic measurements of the orophaynx. Oral Surg Oral Med Oral Pathol Oral Radiol Endod. 2008;106(4):563-70. DOI:10.1016/j.tripleo.2008.03.032

28. El H, Palomo JM. Measuring the airway in 3 dimensions: a reliability and accuracy study. Am J Orthod Dentofac Orthop. 2010;137(4):S50-e1. DOI:10.1016/j.ajodo.2009.11.010

29. Jeans W, Fernando D, Maw A, Leighton B. A longitudinal study of the growth of the nasopharynx and its contents in normal children. Br J Radiol. 1981;54(638):11721. DOI:10.1259/0007-1285-54-638-117

30. Kollias I, Krogstad O. Adult craniofacial and pharyngeal changes-a longitudinal cephalometric study between 22 and 42 years of age. Part II: morphological uvulo-glossopharyngeal changes. Eur J Orthod. 1999;21(4):345-55. DOI:10.1093/ejo/21.4.345 